\title{
Drawing ellipses in water: evidence for dynamic constraints in the relation between velocity and path curvature
}

\author{
Giovanna Catavitello $^{1,2} \cdot$ Yuri P. Ivanenko ${ }^{1}$ Francesco Lacquaniti ${ }^{1,2,3}$. \\ Paolo Viviani ${ }^{1,2}$
}

Received: 8 October 2015 / Accepted: 20 January 2016

(C) Springer-Verlag Berlin Heidelberg 2016

\begin{abstract}
Several types of continuous human movements comply with the so-called Two-Thirds Power Law $(2 / 3-\mathrm{PL})$ stating that velocity $(V)$ is a power function of the radius of curvature $(R)$ of the endpoint trajectory. The origin of the 2/3-PL has been the object of much debate. An experiment investigated further this issue by comparing two-dimensional drawing movements performed in air and water. In both conditions, participants traced continuously quasi-elliptic trajectories (period $T=1.5 \mathrm{~s}$ ). Other experimental factors were the movement plane (horizontal/vertical), and whether the movement was performed free-hand, or by following the edge of a template. In all cases a power function provided a good approximation to the $V-R$ relation. The main result was that the exponent of the power function in water was significantly smaller than in air. This appears incompatible with the idea that the power relationship depends only on kinematic constraints and suggests a significant contribution of dynamic factors. We argue that a satisfactory explanation of the observed behavior must take into account the interplay between the properties of the central motor commands and the visco-elastic nature of the mechanical plant that implements the commands.
\end{abstract}

Yuri P. Ivanenko

y.ivanenko@hsantalucia.it

1 Laboratory of Neuromotor Physiology, Santa Lucia Foundation, via Ardeatina, 306-00179 Rome, Italy

2 Centre of Space BioMedicine, University of Rome Tor Vergata, 00133 Rome, Italy

3 Department of Systems Medicine, University of Rome Tor Vergata, 00133 Rome, Italy
Keywords Drawing movements · Visco-elastic forces . Endpoint trajectory $\cdot$ Kinematic constraints $\cdot$ Two-thirds power law

\section{Introduction}

At the International Congress of Psychology held in Chicago in 1908 Binet presented a communication entitled "Quelques observations sur les movements d'écriture" [Some remarks on writing movements]. Using an Edison pen that burned the paper at a fixed rate with electric sparks, he had observed that, where the writing trace was more curved, the marks were more closely spaced, indicating a decreased velocity in those regions (Binet and Courtier 1893). A similar observation was independently reported by Jack (1894). Almost 90 years later Viviani and Terzuolo (1982) provided a more detailed description of this phenomenon in drawing and handwriting movements. They suggested that the tangential velocity $V(t)$ of the pen's tip is approximately proportional to the radius of curvature $R(t)$ of the trace. Revising this suggestion, Lacquaniti et al. (1983) showed that $V(t)$ is approximately proportional to the cubic root of $R(t)$. Because angular velocity $A(t)=V(t) / R(t)$ and curvature $C(t)=1 / R(t)$, the same relation can be expressed equivalently as $A(t)=\mathrm{KC}(t)^{2 / 3}$, this latter description being usually referred to as Two-Thirds Power Law (henceforth 2/3-PL).

Since the original demonstration, it has been reported that the law also applies to different types of motion, such as tongue movements during speech (Tasko and Westbury 2002; Perrier and Fuchs 2008), locomotive trajectories (Vieilledent et al. 2001; Ivanenko et al. 2002; Hicheur et al. 2005; Pham et al. 2007), and smooth eye movements (de'Sperati and Viviani 1997). It was also found that the 
2/3-PL evolves with age during childhood (Sciaky et al. 1987; Viviani and Schneider 1991) and interacts in subtle ways with the spontaneous tendency to modulate the average velocity as a function of movement size (Viviani and McCollum 1983; Viviani and Cenzato 1985; Viviani et al. 2009).

In hand drawing, deviations from the 2/3-PL occur at inflection points where the prescribed tangential velocity would become infinite, but they also occur for some movements without inflection points (Wann et al. 1988; Schaal and Sternad 2001; Flash and Handzel 2007; Huh and Sejnowski 2015). Thus, a recent study confirmed the 2/3-PL for drawing ellipses, but showed a spectrum of power laws with exponents covering a wide range for simple movements that can be characterized by a single angular frequency (for a closed figure, the number of curvature oscillations over one full rotation, Huh and Sejnowski 2015).

The origin of the connection between geometrical and kinematical aspects of voluntary endpoint movements has been the object of much debate. Major emphasis has been given to the idea that kinematic constraints in trajectory planning and control underlie the power law. In this vein, it has been proposed that the power law arises from optimal control involving the minimization of a kinematic cost functions, such as the total variance of endpoint position (Harris and Wolpert 1998) or the total squared jerkcost (jerk, time derivative of the acceleration, Flash and Hogan 1985; Wann et al. 1988; Viviani and Flash 1995; Todorov and Jordan 1998; Richardson and Flash 2002; Huh and Sejnowski 2015). In particular, the most recent model of minimum-jerk is formulated in a local moving reference frame and predicts both the 2/3-PL for ellipses as well as the range of the exponent values of the power law observed with more complex shapes (Huh and Sejnowski 2015).

Another kinematic constraint that can predict the power law is provided by the principle of constant equi-affine speed (Pollick and Sapiro 1997; Flash and Handzel 2007; Pollick et al. 2009) or a combination of Euclidean, affine, and equi-affine geometries (Bennequin et al. 2009). Interestingly, the principle of constant equi-affine speed leads to a generalization of the power law to three-dimensional (3D) movements (Maoz et al. 2009; Pollick et al. 2009). Still another kinematic constraint that has been shown to be compatible with the 2/3-PL for ellipses is represented by the composition of simple harmonic oscillations with the same frequency and a phase offset, either involving the Cartesian coordinates of endpoint motion (Lacquaniti et al. 1983) or the angular coordinates of upper limb segments motion (Soechting and Terzuolo 1986; Schaal and
Sternad 2001; Dounskaia 2007). Kinematic constraints are also defined by neurons in motor cortex whose population activity encodes the instantaneous velocity vector of drawing movements, so that the coupling between velocity and curvature is observable at the level of the central representation of the trajectory (Schwartz 1994).

On the other hand, it has been argued that dynamic constraints may contribute to the origin of the 2/3-PL, in addition to kinematic constraints (Wann et al. 1988; Gribble and Ostry 1996; Harris and Wolpert 1998). Thus, Wann et al. (1988) proposed a jerk-cost of a mass-spring-dashpot system. In a similar vein, Gribble and Ostry (1996) showed that the 2/3-PL can emerge in a multi-jointed system endowed with visco-elastic muscle properties, which is driven by a control signal specifying a constant speed trajectory. Instead, the minimum position-variance model assumes that the control signals are corrupted by noise whose variance increases with the size of the control signal, another dynamic factor (Harris and Wolpert 1998). Dynamic constraints may also arise as a corollary of endpoint force encoding by neurons in motor cortex (Georgopoulos et al. 1992).

Distinguishing between dynamic and kinematic factors at the origin of the relation between velocity and curvature has proven difficult so far, because all models reviewed above predict a 2/3-PL for drawing ellipses under normal conditions. One strategy to reveal dynamic contributions on top of the kinematic constraints is to compare drawing movements performed in two media of different viscosities, such as water versus air. Because the higher viscosity of water results in higher resistive forces proportional to speed, the overall dynamic behavior will change in water, but kinematics may or may not be affected depending on whether dynamic or kinematic constraints predominate in endpoint control. Specifically, if the velocity-curvature relationship only depended on kinematic constraints, the 2/3-PL should hold in water just as in air. If instead dynamic factors played an important role, the velocity-curvature relationship should change as a function of medium viscosity.

Here we tested these alternatives by asking participants to draw ellipses in air and in water. The test involved both free-hand drawing of ellipses and movements guided by a quasi-elliptic template formed by joining smoothly two pairs of circular arcs with different radii. The template condition was designed to address in a controlled manner the relation between the geometry of the trajectory and the velocity of execution. Specifically, we wanted to investigate how velocity changed across the four points within a movement cycle in which the radius of curvature changed abruptly. 


\section{Methods}

\section{Participants}

Ten individuals ( 6 men, 4 women) volunteered for the experiment. All participants but one were right-handed (as assessed by a short questionnaire based on the Edinburgh scale) and had no neurological disorders. Participants gave written informed consent to procedures approved by the Institutional Review Board of Santa Lucia Foundation, in conformity with the Declaration of Helsinki on the use of human subjects in research. They were otherwise unaware of the purpose of the experiments.

\section{Apparatus and protocol}

The experiments were run in a laboratory space with artificial illumination. Participants lied on a medical examination bed in a prone position close enough to the bed edge to allow the dominant arm to move freely in the underneath space. Aligned with the bed there was a Plexiglas tank ( $L: 100 \mathrm{~cm} ; W: 45 \mathrm{~cm} ; H: 50 \mathrm{~cm})$ that could be filled with water kept at a roughly constant temperature of $20{ }^{\circ} \mathrm{C}$ (Fig. 1a). The height of the tank with respect to the bed top was adjusted so that with a partially flexed arm the hand of the participant could reach comfortably either the bottom of the tank or the lateral wall nearest to the bed. The general task was to draw closed trajectories continuously on these two surfaces using the pen of a digitizing tablet (Wacom Intuos2; accuracy: $0.25 \mathrm{~mm}$, resolution: 100 lines $/ \mathrm{mm}$, sampling rate: 200 samples/s). The tablet was positioned outside the tank, either below the bottom or outside the lateral wall. In either case, the thickness of the tank walls was smaller than the maximum allowed distance between the pen and the tablet $(6 \mathrm{~mm})$. Thus, movement was recorded accurately even though the electromagnetic pen was not directly in contact with the tablet surface. The tempo of the movement was dictated by an electronic metronome ( 1 complete cycle: $1.5 \mathrm{~s}$ ).

Three factors defined the experimental plan. The first factor was the medium in which movements were performed, namely air $(A)$ or water $(W)$. In condition $A$, the tank was empty and, in spite of the somewhat unusual prone posture, movements were not much different qualitatively from those required to draw on the floor or on a wall. In condition $W$, the tank was almost completely filled, the level of the water reaching roughly the mid-point of the upper arm. The velocity of the movement was not sufficient to generate appreciable turbulence in the water, but high enough to increase substantially the resistive viscous forces with respect to condition $A$. The second factor was the drawing modality. In one condition ( $T$ : template), the movement was guided by asking the participant to follow

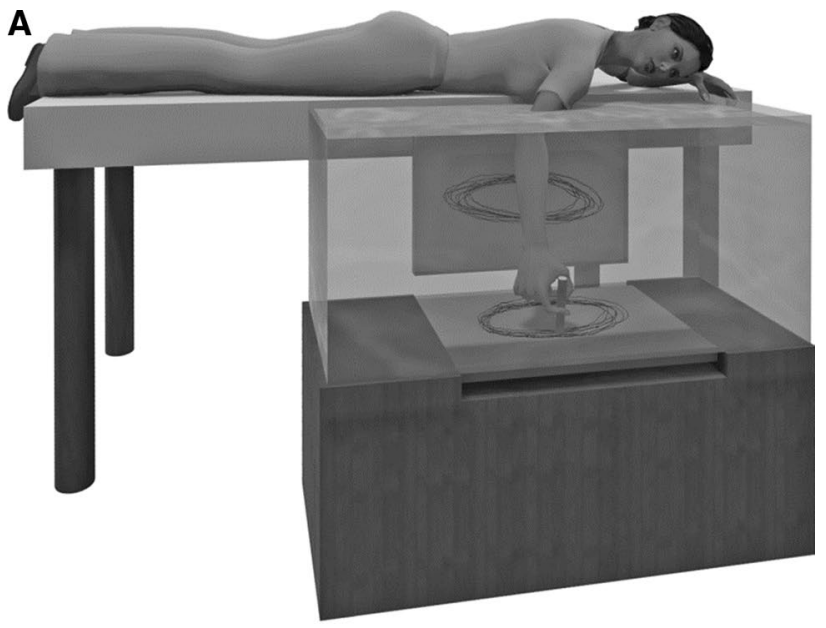

B

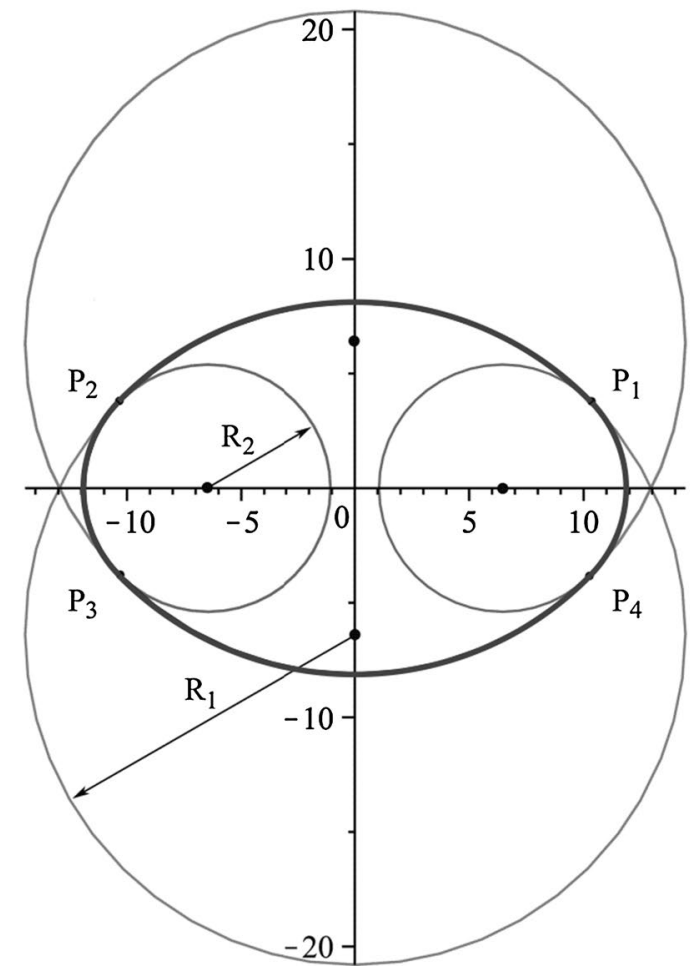

Fig. 1 a Experimental setup. The drawing plane could be on the bottom of the tank (condition "Horizontal": $H$ ) or on the side wall closer to the participant (condition "Vertical": $V$ ). Participants drew freely (condition "Free": $F$ ) or following the inner edge of a plastic template (condition "Template": $T$ ). The tank could be empty (condition "Air": A) or filled with water (condition "Water": W). b Geometrical description of the template used in the experiments. The inner edge of the template (heavy line) resulted from joining smoothly two pairs of arc of circle with different radii (light lines)

with the tip of the pen the inner edge of a Plexiglas template cut by a high-precision computer-controlled mill machine (Fig. 1b). The edge was perfectly smooth and did not generate appreciable frictional forces. The shape of the template resembled an ellipse, but was actually realized 
by joining smoothly two pairs of circular arcs with radius $R_{1}=14.44 \mathrm{~cm}$ and $R_{2}=5.39 \mathrm{~cm}$, respectively. The long and short axes of the template were 23.7 and $16.2 \mathrm{~cm}$, respectively. The long axis was aligned with the body of the participant. The perimeter was $62.64 \mathrm{~cm}$. In a second condition $(F$ : free), participants were instructed to draw freely elliptic trajectories with approximately the same dimensions of the template and the same orientation with respect to the body. The third factor was the orientation of the drawing plane ( $H$ : Horizontal, i.e., the tank bottom; $V$ : Vertical, i.e., the tank wall). All levels of the factors were crossed in a complete factorial design (8 conditions). Factors were blocked. Half of the participants were tested first in the $A$ condition and then in the $W$ condition. For the other half the order was inverted. Within each $A / W$ condition, the four remaining combinations $(T-H, T-V, F-H$, $F-V)$ were administered in a pseudo-random order. Three identical trials were recorded successively for each of the 8 combinations. The recording started after the participants had completed a few warm-up cycles used to synchronize the movement with the metronome. Each trial lasted $20 \mathrm{~s}$ and included at least 12 complete movement cycles.

\section{Data processing}

After filtering the $(20 \mathrm{~s} \times 200 \mathrm{samples} / \mathrm{s}=4000)$ displacement samples (fourth-order, zero-phase lag Butterworth filter; cut-off frequency: $8 \mathrm{~Hz}$ ), we characterized the trajectories in the $F$ condition by three measures. The average perimeter $P$ was estimated by integrating the instantaneous velocity over all complete cycles of the movement. The eccentricity $\Sigma$ was defined as $\Sigma=\sqrt{1-\left(A_{y} / A_{x}\right)^{2}}$ where $A_{y}$ and $A_{x}$ are the minor and major semi-axis of the trajectory estimated by the formulae:

$A_{x}, A_{y}=\sqrt{S_{x}+S_{y} \pm \sqrt{\left(S_{x}+S_{y}\right)^{2}-4 S_{x} S_{y}\left(1-\frac{C_{x y}}{\sqrt{S_{x} S_{y}}}\right)}}$

where $S_{x}, S_{y}$ and $C_{x y}$ are the variances and the covariance, respectively, of the samples $\left(x_{i}, y_{i}\right)$ of all complete cycles. The angles $\Omega_{1}$ and $\Omega_{2}$ of the axes of the trajectory with respect to the direction of the body axis were estimated by the formulae:

$\operatorname{tg}\left(\Omega_{1}\right), \operatorname{tg}\left(\Omega_{2}\right)=\frac{S_{y}-S_{x}}{2 C_{x y}} \pm \sqrt{\left(\frac{S_{y}-S_{x}}{2 C_{x y}}\right)^{2}+1}$

From the samples $\left(x_{i}, y_{i}\right)$ we also computed the tangential velocity $V(t)=\sqrt{(\mathrm{d} x / \mathrm{d} t)^{2}+(\mathrm{d} y / \mathrm{d} t)^{2}}$ and the radius of curvature $R(t)=\frac{V(t)^{3}}{\left|\left(\mathrm{~d}^{2} x / \mathrm{d} t^{2}\right)(\mathrm{d} y / \mathrm{d} t)-\left(\mathrm{d}^{2} y / \mathrm{d} t^{2}\right)(\mathrm{d} x / \mathrm{d} t)\right|}$ of the

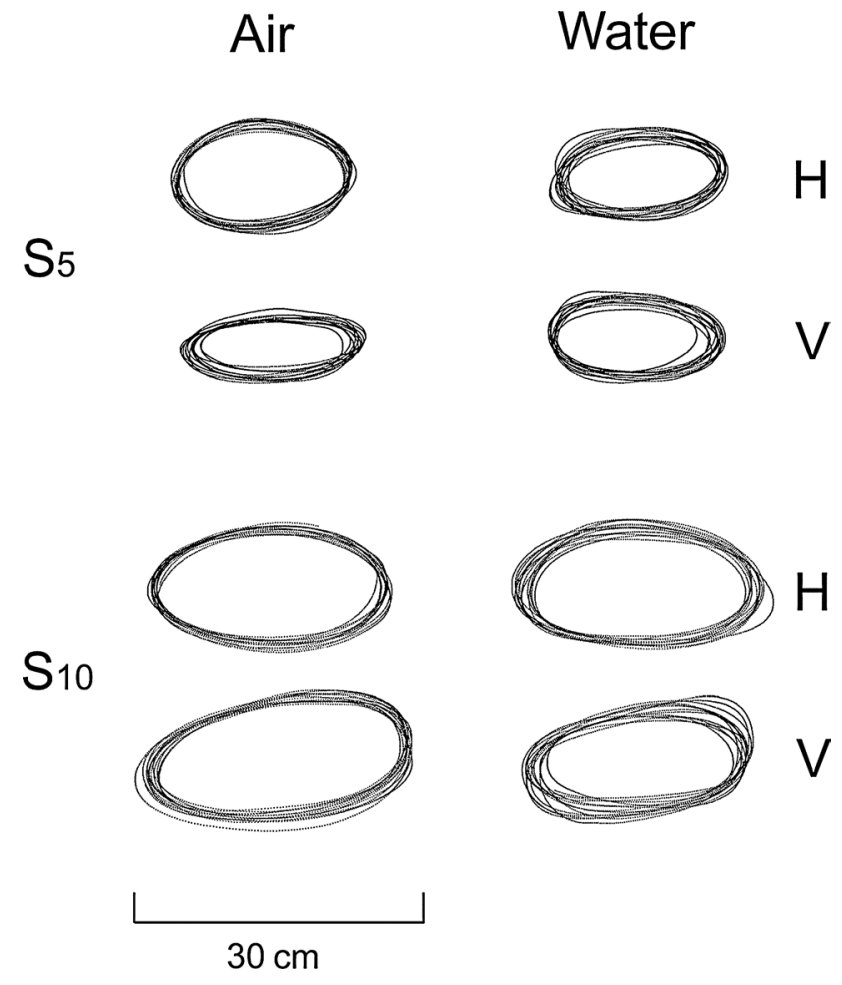

Fig. 2 Free-hand drawing. Typical examples in two participants $\left(S_{5}\right.$, $S_{10}$ ) of one complete recording (20 s) of the drawing movement for each combination of orientation $(H / V)$ and medium (Air/Water)

trajectory. By assuming that $V(t)$ and $R(t)$ are related by the 2/3-PL $V(t)=K R(t)^{1-\beta}(\beta \approx 2 / 3)$, we estimated the gain factor $K$ and the exponent $\beta$ by a correlation analysis of the scatter diagram $[\log R(t), \log V(t)]$. Specifically, let $[y=a$ $x+b$ ] be the equation of the $V(t)$-over- $R(t)$ regression through the scatter. Then, $K=e^{b}$ and $\beta=1-a$. Unless otherwise stated, statistical significance of the experimental factors was tested with the general linear model for repeated measures applying the Greenhouse-Geisser correction for non-sphericity.

\section{Results}

When movements were guided (condition $T$ ), the trajectories followed quite accurately the edge of the template. With rare exceptions when the pen deviated slightly from the imposed trajectory, the perimeter of the trace coincided with that of the template. In the free-hand condition $(F)$, the trajectories were generally elliptical. Figure 2 illustrates with examples from two participants the trajectories drawn freely in the four conditions $A-H, A-V, W-H$, and $W-V$. In a few cases, the cycle-by-cycle variability was higher than in these examples, particularly when drawing on the tank wall (condition $V$ ). Table 1 summarizes the estimated 
Table 1 Perimeter $P$, eccentricity $\Sigma$, and angle of the major axis $\operatorname{tg}(\Omega)$ of the movements in the free-hand drawing of ellipses condition in the horizontal and vertical plane

Fig. 3 Power law relation between radius of curvature $(R)$ and velocity $(V)$. Typical examples in one participant of the relation (log/log scales) between $R$ and $V$ in all 8 experimental conditions ( $H$ : Horizontal, $V$ : Vertical, $F$ : Free-hand, $T$ : template, $A$ : Air, $W$ : Water). Scatterplots represent one recording. A few outliers in condition $F(R<2 \mathrm{~cm}, R>25 \mathrm{~cm})$ are not reported. Light lines 0.95 confidence ellipses and axes of orthogonal regression. Slopes of the scatterplots (see Table 2) were estimated from the $\log (V)$ over $\log (R)$ linear regression (not shown)

\begin{tabular}{llllllll}
\hline & Horizontal & & & & Vertical & & \\
\cline { 2 - 3 } \cline { 7 - 8 } & $P(\mathrm{~cm})$ & $\Sigma$ & $\operatorname{tg}(\Omega)$ & & $P(\mathrm{~cm})$ & & \multicolumn{1}{c}{$\Sigma(\Omega)$} \\
\hline Air & 55.52 & 0.880 & 0.035 & & 56.84 & 0.803 & -0.053 \\
Water & 54.82 & 0.807 & 0.034 & & 54.37 & 0.755 & -0.108 \\
\hline
\end{tabular}
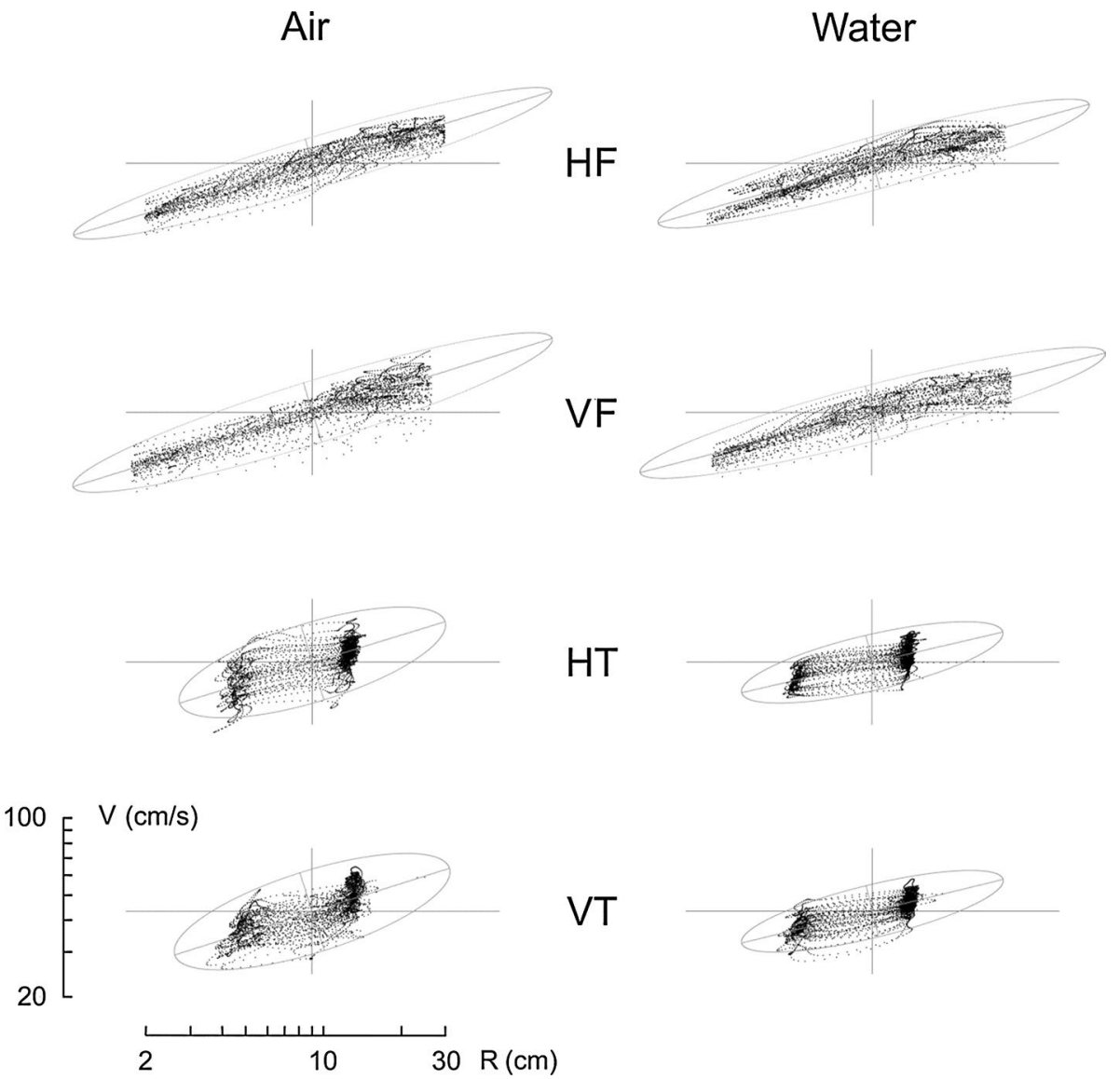

geometrical parameters (perimeter, eccentricity, angle of the major axis) of the movements in the $F$ condition. The perimeter of the trajectory depended neither on the medium (air vs water) in which the movement was performed $(2[A / W]: F(9,1)=0.643, P=0.443)$ nor on the orientation of the drawing plane $(2[H / V]: F(9,1)=0.890, P=0.370)$. Moreover, there was no interaction of the medium with either the orientation $(2[A / W] \times 2[H / V]:[F(9,1)=0.001$, $P=0.981)$ or the modality of execution $(2[A / W] \times 2[F / T]$ : $F(9,1)=0.022, P=0.886)$. The perimeter was instead significantly larger when drawing freely than when following the template $(2[F / T]: F(9,1)=13.563, P=0.005)$. The eccentricity $\Sigma$ of the trajectory was higher in the free-hand than in the template condition $(2[F / T]: F(9,1)=24.305$, $P=0.001)$ and also higher in air than in water $(2[A / W]$ : $F(9,1)=9.659, P=0.013)$. Orientation had a marginal effect $(2[H / V]: F=4.196, P=0.071)$, but interacted significantly with the modality $(2[H / V] \times 2[F / T]$ :
$F(9,1)=14.078, P=0.005)$. The major axis of the trajectory deviated with respect to the body axis. By convention, the slope $\operatorname{tg}(\Omega)$ of the axis was negative when the rotation was clockwise (i.e., in the direction of the movement) and positive when the rotation was counterclockwise. The average slope over all participants and trials was HA: 0.035, HW: 0.033, VA: 0.053, VW: 0.108.

Figure 3 illustrates for one representative participant the analysis of the relationship between tangential velocity and radius of curvature. For each combination of the experimental factors, the Figure shows the scatter diagrams of the samples recorded in one of the three repetitions and the $95 \%$ ellipses of confidence with the associated axes (light lines). In the free-hand $(F)$ condition, we excluded for the analysis the samples for which either $R>25 \mathrm{~cm}$, corresponding to short segments of the trajectory that included inflections, or $R<2 \mathrm{~cm}$, corresponding to occasional lack of smoothness in the movement. No more that 
Table 2 Individual means for each subject (over 3 repetitions) of the slope of the linear regression of $\log (\mathrm{V}(\mathrm{t}))$ over $\log (R(t))$

\begin{tabular}{lllllllll}
\hline & HFA & HFW & VFA & VFW & HTA & HTW & VTA & VTW \\
\hline S01 & 0.30704 & 0.18036 & 0.24869 & 0.13764 & 0.32628 & 0.15705 & 0.28160 & 0.21124 \\
S02 & 0.31839 & 0.21832 & 0.21851 & 0.23205 & 0.46068 & 0.37322 & 0.31336 & 0.27584 \\
S03 & 0.33329 & 0.30126 & 0.29987 & 0.26323 & 0.13910 & 0.26162 & 0.20510 & 0.23258 \\
S04 & 0.30139 & 0.26407 & 0.30862 & 0.31474 & 0.43957 & 0.30433 & 0.26274 & 0.38746 \\
S05 & 0.27538 & 0.30676 & 0.31149 & 0.26333 & 0.37275 & 0.31776 & 0.37729 & 0.33923 \\
S06 & 0.31309 & 0.26027 & 0.28009 & 0.23546 & 0.29381 & 0.31901 & 0.32433 & 0.23064 \\
S07 & 0.30693 & 0.31404 & 0.30124 & 0.26664 & 0.29878 & 0.24036 & 0.36219 & 0.26411 \\
S08 & 0.31003 & 0.25753 & 0.29358 & 0.25558 & 0.27532 & 0.26401 & 0.30875 & 0.27723 \\
S09 & 0.34601 & 0.30608 & 0.24588 & 0.31223 & 0.35848 & 0.32093 & 0.29495 & 0.29060 \\
S10 & 0.35338 & 0.29116 & 0.33438 & 0.31088 & 0.43697 & 0.30231 & 0.27391 & 0.32281 \\
Av. & 0.31649 & 0.26998 & 0.34017 & 0.28606 & 0.28423 & 0.25917 & 0.30042 & 0.28317 \\
SD & 0.02278 & 0.04347 & 0.09652 & 0.05898 & 0.03581 & 0.05243 & 0.04958 & 0.05435 \\
\hline
\end{tabular}

The average and standard deviation over all subjects are reported in the last 2 rows. Acronyms for the columns are defined in the text. For instance, HFA stands for movements in the Horizontal plane, Free-hand condition, in Air

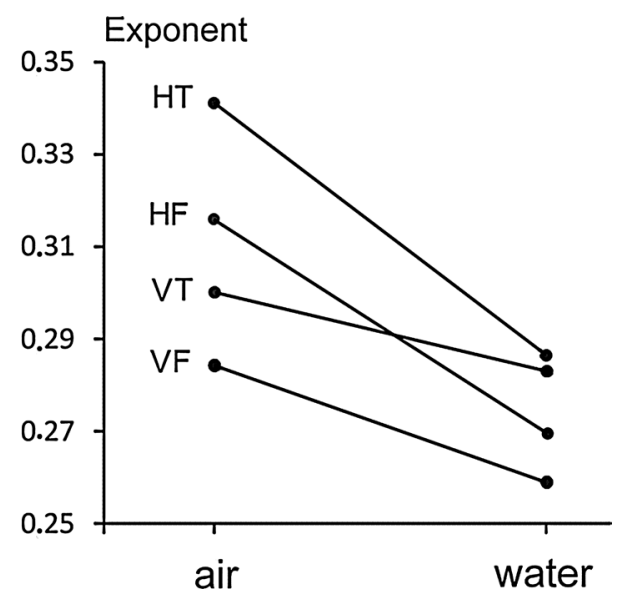

Fig. 4 Effects of the medium on the exponent of the power law. Averages over all repetitions and participants. $H$ horizontal; $V$ vertical; $F$ free-hand; $T$ template

$4 \%$ of the samples were discarded. The selection was not necessary in the template $(T)$ condition where the radius was constrained. The dispersion of the data points along the vertical (velocity) axis was greater in the $T$ that in the $F$ condition. Inspection of the individual cycles in condition $T$ revealed that, although the imposed duration of one cycle was always respected accurately, participants did not consistently complete in equal times the two halves of the trajectory extending from the opposite points of minimum curvature.

Table 2 reports for all conditions the individual means over three repetitions of the slope of the linear regression of $\log (V(t))$ over $\log (R(t))$, i.e., the estimated exponent $1-\beta$ in the 2/3-PL (see Data Processing). With few exceptions, for both movement orientations $(H / V)$ and both movement modality $(F / T)$, in all participants the exponent $1-\beta$ was smaller in water than in air. At the population level (Fig. 4), this main effect was highly significant $(2[A / W]$ : $F(9,1)=9.308, P=0.014$, Partial Eta Squared $=0.508$ ). The orientation also affected the exponent, which was lower in the $H$ than in the $V$ condition $(2[H / V]: F(9,1)=5.604$, $P=0.042$, Partial Eta Squared $=0.384$ ). No interaction between factors was significant.

\section{Moving along the template}

Movements along the template edge crossed four points placed symmetrically along the trajectory (see Fig. 1b) where the radius of curvature switched abruptly between a large $\left(R_{1}\right)$ and a small $\left(R_{2}\right)$ value. The instantaneous velocity change predicted by the $2 / 3$-PL at these points is physically impossible because it would require an infinite force. The template was designed to investigate how the actual velocity departs from this ideal prediction. Figure 5a shows all excerpts $(450 \mathrm{~ms}$ ) of the velocity around a radius change recorded in a typical HA trial for one participant. Panel B summarizes for each experimental condition the results for all trials and participants. There were quantitative differences between the transitions $R_{1} \rightarrow R_{2}$ and $R_{2} \rightarrow R_{1}$. Averaged across conditions, transition times were shorter (318 ms) for $R_{2} \rightarrow R_{1}$ than for $R_{1} \rightarrow R_{2}$ (392 ms). However, the average slopes of the velocity measured over the $30 \mathrm{~ms}$ after the radius change were not statistically different between transitions $\left(2\left[R_{1} \rightarrow R_{2}, R_{2} \rightarrow R_{1}\right] \times 2[H\right.$, $V] \times 2[A, W], F(1,9)=0.602, P=0.458)$. A significant slope difference emerged instead from the contrast HA-HW (pooling over transitions and trials, 2-tailed $t$ test for paired samples, $T(9)=2.431, P=0.038$ ), showing that velocity changed faster in air than in water. Decreasing 
Fig. 5 Movements with template. Time course of the velocity across sudden changes (arrows) of the radius of curvature (straight lines, arbitrary scales). a Velocity profiles for one representative trial (condition HA) in one participant (light lines). Transitions for all cycles within the $20 \mathrm{~s}$ recording. The data for the two transitions from large to small radius (upper graphs) and for the two transitions from low to high radius (lower graphs) are pooled separately. Average velocity profiles (heavy lines) for the trial are also shown. The average velocity $\left(V_{m}\right)$ was very close to the value predicted by the ratio of the template perimeter $(P=62.64 \mathrm{~cm})$ to the imposed tempo $(T=1.5 \mathrm{~s})$. $\mathbf{b}$ Average (black line) and $95 \%$ confidence intervals (blue lines) across participants. $H / V$ Trials in the horizontal/vertical plane; $A / W$ Trials in air/water. Radii are in arbitrary scale
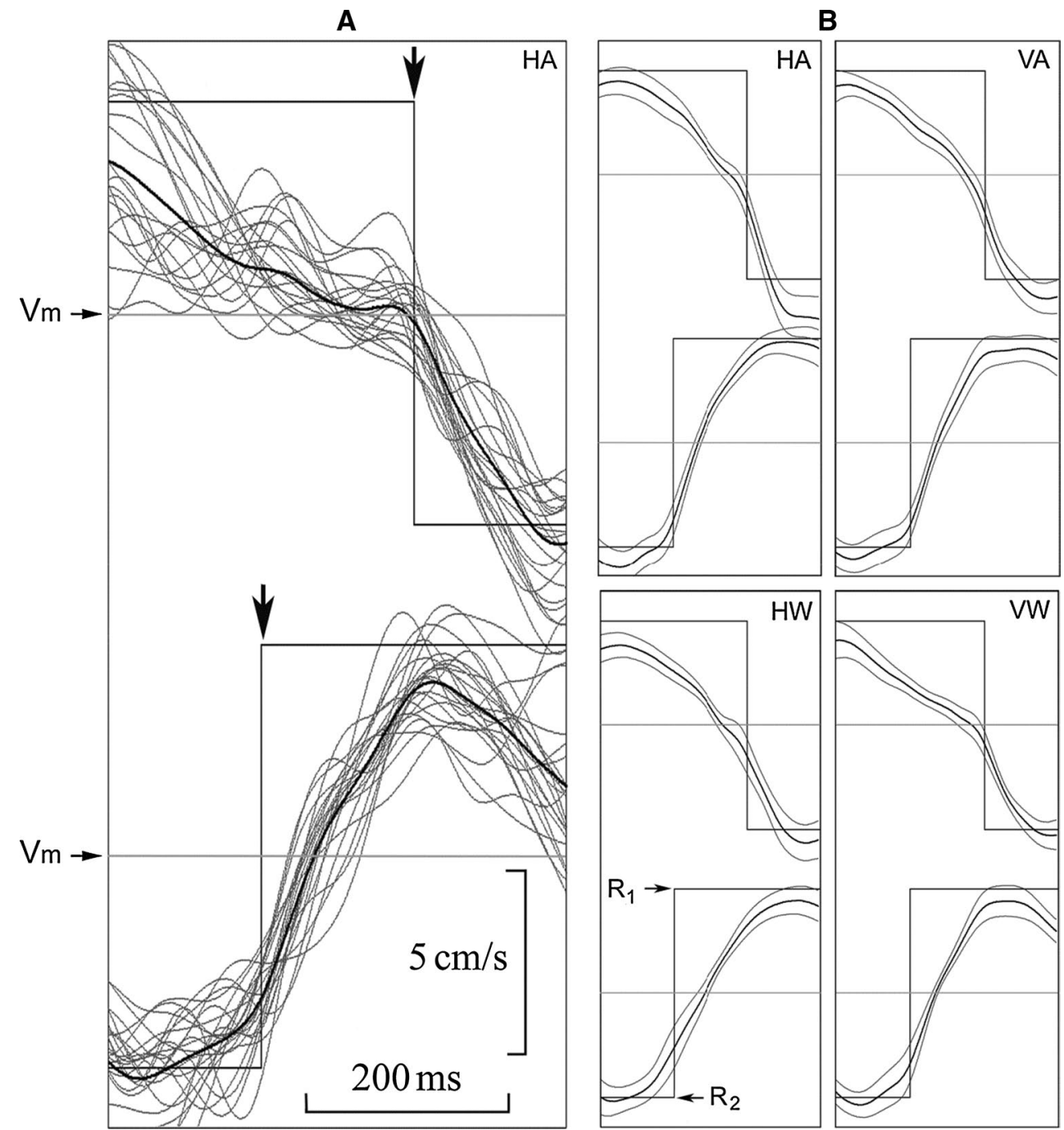

velocities $\left(R_{1} \rightarrow R_{2}\right)$ crossed their average value $\left(V_{\mathrm{m}}\right.$, light lines) before the change in radius, whereas increasing velocities $\left(R_{2} \rightarrow R_{1}\right)$ crossed after the change in radius. First, we considered the time interval between crossing and transitions by keeping separate the four transitions points within a cycle. The analysis (4[Transition] $\times 2[H$, $V] \times 2[A, W]$ ) showed that only the direction of the transition was a significant factor. A post hoc analysis after pooling the two pairs of transitions in the same direction confirmed the highly significant difference between increasing and decreasing velocities $\left(2\left[R_{1} \rightarrow R_{2}, R_{2} \rightarrow R_{1}\right] \times 2[H\right.$, $V] \times 2[A, W], F(1,29)=71.956, P<0.001)$. Averaged over participants and trials, the time interval between ascending and descending crossing was HA: $108 \mathrm{~ms}$, HW: $83 \mathrm{~ms}$, VA: $110 \mathrm{~ms}$, and VW: $104 \mathrm{~ms}$. Finally, one qualitative difference was present in all trials and all four conditions, namely velocity changes were more uniform when switching from low to large radii than in the opposite direction.

\section{Discussion}

We have demonstrated that a violation of the 2/3-PL occurs when drawing movements are performed in a medium (water) that opposes a higher viscous resistance than air, where they are normally executed. Specifically, the exponent of the power law is significantly smaller in water than in air, indicating a tendency for velocity to be less dependent on the curvature of the trajectory than it is in air. Violations of the 2/3-PL have emerged previously from the analysis of air-stepping (Ivanenko et al. 2002), articulatory speech (Perrier and Fuchs 2008), and some 3D movements (Schaal and Sternad 2001). It has also been reported that the law is inaccurate in the case of slow movements and movements with strongly non-symmetrical velocity profiles (Wann et al. 1988). Recently, it has been shown that the exponent of the power law depends on the drawn 
shape, being a function of the angular frequency (Huh and Sejnowski 2015).

The violation reported here does not fall into any of the above categories. First, the tested movements were prototypically smooth and oscillatory, both in air and water. Moreover, their average velocity was well within the range of common voluntary gestures. Notably, the shape of the drawn figures was not significantly affected by whether the movements were performed in air or water. Finally, the departure from the 2/3-PL documented here manifested itself only in the value of the exponent.

Another remarkable departure from the 2/3-PL here emerged from the analysis of the kinematics when the movement was guided by the edge of the template. By zooming on a 450-ms time window encompassing a sudden transition from segments of trajectory with different radii, we observed significant differences in the corresponding velocity changes. Some characteristics of these velocity changes, such as the crossing of the average value before the radius change in the transition $R_{1} \rightarrow R_{2}$ (Fig. 5), may reflect an anticipatory control of path trajectory (Tramper and Flanders 2013). However, it is unclear why the reverse transition $R_{2} \rightarrow R_{1}$ did not involve a similar anticipation. A more in-depth testing of trajectories with predictable discontinuities would be required to clarify this point.

In its simplest formulation, the $2 / 3$-PL has limitations due to physics. The law cannot deal with inflection points where the prescribed velocity would become infinite. Moreover, it cannot deal with discontinuities in the radius of curvature where the prescribed acceleration would become infinite. Not surprisingly the actual velocity variations were in fact continuous. The first limitation can be allayed by modifying the original form of the law (cf. Viviani and Stucchi 1992). The second one, instead, calls for a more extensive reformulation whereby purely kinematical concepts are replaced by dynamical ones. Indeed, if the velocity-curvature relationship only depended on kinematic constraints, the 2/3-PL should hold in water just as in air. Thus, execution of hand movements involving feedback-regulated geometry and speed should keep the 2/3-PL invariant, regardless of external resistive forces. Instead, the observation that the velocity-curvature relationship systematically changed as a function of medium viscosity argues for a critical role of dynamic factors.

Several types of dynamic factors presumably concur to account for the observed results. First, the musculoskeletal apparatus behaves as a mass-spring-dashpot system and acts as a low-pass filter on the neural signals. In this regards, a previous modeling study of drawing in air showed that even a crude neural command signal that specifies a constant speed trajectory, once filtered by the musculoskeletal apparatus, results in a velocity-curvature relationship consistent with the 2/3-PL (Gribble and Ostry
1996). The net impedance of the musculoskeletal apparatus combines with that of the medium within which the movement is performed. In addition, velocity-dependent dynamics is presumably incorporated in the central commands. In this regards, it has been shown that the time-varying activity of neural populations in motor cortex reflects the instantaneous velocity vector of drawing movements (Schwartz 1994). Moreover, the magnitude of the population vector obeys the 2/3-PL, while the direction of the population vector matches the instantaneous movement direction (Schwartz 1994). It has further been hypothesized that the coupling between speed and curvature, also observable at the level of the neural representation of the trajectory, results from the neural transformation of the intended direction which is an inherently time-consuming process, the duration of which increases with the angle of rotation while steering a drawing movement (Pellizzer 1997).

A velocity-curvature covariance is predicted by models of optimal control maximizing a smoothness cost, such as the total squared jerk-cost (Flash and Hogan 1985; Wann et al. 1988; Viviani and Flash 1995; Todorov and Jordan 1998; Richardson and Flash 2002; Huh and Sejnowski 2015). In particular, it has been shown that replacing the assumption of a Newtonian point-mass in a minimum-jerk model with a more realistic visco-elastic body accounts well for both the typical 2/3-PL as well as departures from the typical behavior under special conditions (Wann et al. 1988). This model assumes that the cost function being minimized includes both an external and an internal jerk function, with the result that changes in overall stiffness and/or viscosity will affect the specific form of the velocity-curvature relationship (Wann et al. 1988). We believe that control laws incorporating dynamic terms, such as the modified minimum-jerk (Wann et al. 1988), minimumenergy (Nelson 1983), or minimum-torque-change (Uno et al. 1989), can account for the changes of the exponent in the velocity-curvature relationship we observed with changes of the medium viscosity.

Acknowledgments The work was supported by the Italian Ministry of University and Research (PRIN Grant 2010MEFNF7_002), Italian Space Agency (COREA Grant 2013-084-R.0) and Horizon 2020 robotics program (ICT-23-2014 under Grant Agreement 644727CogIMon). We wish to thank Roberto Catavitello for designing and realizing the tank used for the experiments.

\section{References}

Bennequin D, Fuchs R, Berthoz A, Flash T (2009) Movement timing and invariance arise from several geometries. PLoS Comput Biol 5:e1000426. doi:10.1371/journal.pcbi.1000426

Binet A, Courtier J (1893) Sur la vitesse des mouvements graphiques. Rev Philos Fr LÉtranger 35:664-671 
de'Sperati C, Viviani P (1997) The relationship between curvature and velocity in two-dimensional smooth pursuit eye movements. J Neurosci 17:3932-3945

Dounskaia N (2007) Kinematic invariants during cyclical arm movements. Biol Cybern 96:147-163. doi:10.1007/ s00422-006-0109-1

Flash T, Handzel AA (2007) Affine differential geometry analysis of human arm movements. Biol Cybern 96:577-601. doi:10.1007/ s00422-007-0145-5

Flash T, Hogan N (1985) The coordination of arm movements: an experimentally confirmed mathematical model. J Neurosci 5:1688-1703

Georgopoulos AP, Ashe J, Smyrnis N, Taira M (1992) The motor cortex and the coding of force. Science 256:1692-1695

Gribble PL, Ostry DJ (1996) Origins of the power law relation between movement velocity and curvature: modeling the effects of muscle mechanics and limb dynamics. J Neurophysiol 76:2853-2860

Harris CM, Wolpert DM (1998) Signal-dependent noise determines motor planning. Nature 394:780-784. doi:10.1038/29528

Hicheur H, Vieilledent S, Richardson MJE et al (2005) Velocity and curvature in human locomotion along complex curved paths: a comparison with hand movements. Exp Brain Res 162:145-154. doi:10.1007/s00221-004-2122-8

Huh D, Sejnowski TJ (2015) Spectrum of power laws for curved hand movements. Proc Natl Acad Sci 112:E3950-E3958. doi:10.1073/ pnas. 1510208112

Ivanenko YP, Grasso R, Macellari V, Lacquaniti F (2002) Two-thirds power law in human locomotion: role of ground contact forces. NeuroReport 13:1171-1174

Jack WR (1894) On the analysis of voluntary muscular movements by certain new instruments. Proc R Soc Lond 57:477-481. doi:10.1098/rspl.1894.0178

Lacquaniti F, Terzuolo C, Viviani P (1983) The law relating the kinematic and figural aspects of drawing movements. Acta Psychol (Amst) 54:115-130

Maoz U, Berthoz A, Flash T (2009) Complex unconstrained threedimensional hand movement and constant equi-affine speed. J Neurophysiol 101:1002-1015. doi:10.1152/jn.90702.2008

Nelson WL (1983) Physical principles for economies of skilled movement. Biol Cybern 46:135-147

Pellizzer G (1997) Transformation of the intended direction of movement during continuous motor trajectories. NeuroReport 8:3447-3452

Perrier P, Fuchs S (2008) Speed-curvature relations in speech production challenge the 1/3 power law. J Neurophysiol 100:11711183. doi: 10.1152 /jn. 01116.2007

Pham Q-C, Hicheur H, Arechavaleta G et al (2007) The formation of trajectories during goal-oriented locomotion in humans. II. A maximum smoothness model. Eur J Neurosci 26:2391-2403. doi:10.1111/j.1460-9568.2007.05835.x

Pollick FE, Sapiro G (1997) Constant affine velocity predicts the 13 power law of planar motion perception and generation. Vision Res 37:347-353. doi:10.1016/S0042-6989(96)00116-2

Pollick FE, Maoz U, Handzel AA et al (2009) Three-dimensional arm movements at constant equi-affine speed. Cortex 45:325-339. doi:10.1016/j.cortex.2008.03.010
Richardson MJE, Flash T (2002) Comparing smooth arm movements with the two-thirds power law and the related segmented-control hypothesis. J Neurosci 22:8201-8211

Schaal S, Sternad D (2001) Origins and violations of the $2 / 3$ power law in rhythmic three-dimensional arm movements. Exp Brain Res 136:60-72

Schwartz AB (1994) Direct cortical representation of drawing. Science 265:540-542

Sciaky R, Lacquaniti F, Terzuolo C, Soechting JF (1987) A note on the kinematics of drawing movements in children. J Mot Behav 19:518-525

Soechting JF, Terzuolo CA (1986) An algorithm for the generation of curvilinear wrist motion in an arbitrary plane in three-dimensional space. Neuroscience 19:1393-1405

Tasko SM, Westbury JR (2002) Defining and measuring speech movement events. J Speech Lang Hear Res 45:127-142. doi:10.1044/1092-4388(2002/010)

Todorov E, Jordan MI (1998) Smoothness maximization along a predefined path accurately predicts the speed profiles of complex arm movements. J Neurophysiol 80:696-714

Tramper JJ, Flanders M (2013) Predictive mechanisms in the control of contour following. Exp Brain Res 227:535-546. doi:10.1007/ s00221-013-3529-x

Uno Y, Kawato M, Suzuki R (1989) Formation and control of optimal trajectory in human multijoint arm movement. Minimum torquechange model. Biol Cybern 61:89-101

Vieilledent S, Kerlirzin Y, Dalbera S, Berthoz A (2001) Relationship between velocity and curvature of a human locomotor trajectory. Neurosci Lett 305:65-69. doi:10.1016/S0304-3940(01)01798-0

Viviani P, Cenzato M (1985) Segmentation and coupling in complex movements. J Exp Psychol Hum Percept Perform 11:828-845

Viviani P, Flash T (1995) Minimum-jerk, two-thirds power law, and isochrony: converging approaches to movement planning. J Exp Psychol Hum Percept Perform 21:32-53

Viviani P, McCollum G (1983) The relation between linear extent and velocity in drawing movements. Neuroscience 10:211-218

Viviani P, Schneider R (1991) A developmental study of the relationship between geometry and kinematics in drawing movements. J Exp Psychol Hum Percept Perform 17:198-218

Viviani P, Stucchi N (1992) Biological movements look uniform: evidence of motor-perceptual interactions. J Exp Psychol Hum Percept Perform 18:603-623

Viviani P, Terzuolo C (1982) Trajectory determines movement dynamics. Neuroscience 7:431-437. doi:10.1016/0306-4522(82)90277-9

Viviani P, Burkhard PR, Chiuvé SC et al (2009) Velocity control in Parkinson's disease: a quantitative analysis of isochrony in scribbling movements. Exp Brain Res 194:259-283. doi:10.1007/ s00221-008-1695-Z

Wann J, Nimmo-Smith I, Wing AM (1988) Relation between velocity and curvature in movement: equivalence and divergence between a power law and a minimum-jerk model. J Exp Psychol Hum Percept Perform 14:622-637 